\title{
CpGPAP: CpG island predictor analysis platform
}

\author{
Li-Yeh Chuang ${ }^{1}$, Cheng-Huei Yang ${ }^{2}$, Ming-Cheng Lin ${ }^{3}$ and Cheng-Hong Yang ${ }^{3,4^{*}}$
}

\begin{abstract}
Background: Genomic islands play an important role in medical, methylation and biological studies. To explore the region, we propose a CpG islands prediction analysis platform for genome sequence exploration (CpGPAP).

Results: CPGPAP is a web-based application that provides a user-friendly interface for predicting CpG islands in genome sequences or in user input sequences. The prediction algorithms supported in CPGPAP include complementary particle swarm optimization (CPSO), a complementary genetic algorithm (CGA) and other methods (CpGPlot, CpGProD and CpGIS) found in the literature. The CpGPAP platform is easy to use and has three main features (1) selection of the prediction algorithm; (2) graphic visualization of results; and (3) application of related tools and dataset downloads. These features allow the user to easily view CpG island results and download the relevant island data. CpGPAP is freely available at http://bio.kuas.edu.tw/CpGPAP/.
\end{abstract}

Conclusions: The platform's supported algorithms (CPSO and CGA) provide a higher sensitivity and a higher correlation coefficient when compared to CpGPlot, CpGProD, CpGIS, and CpGcluster over an entire chromosome.

\section{Background}

CpG islands are stretches of typically unmethylated DNA with a high content of the two nucleic acids Cytosine $(C)$ and Guanine (G), i.e., a high CG content relative to the bulk DNA. In typical mammalian genomes, many $\mathrm{CpG}$ islands (about 40\%) are found in the promoter region. Of the estimated 45,000 CpG islands in the human genome, the overwhelming majority is found at the 5' end of genes. Identification and cloning of these $\mathrm{CpG}$ islands has proven very useful for finding and isolating genes. The CpG sites in the CpG islands of promoters are unmethylated if genes are expressed. It has thus been speculated that methylation of these sites plays a crucial role in gene expression [1].

CpG islands were originally identified by Tykocinski and Max as small areas that contain the restriction enzyme HpaII in the genome and were thus called HpaII Tiny Fragment (HTF) islands [2]. Gardiner-Garden and Frommer (GGF) defined GpG islands as a DNA sequence with a length exceeding $200 \mathrm{bp}$, a GC content in that region of greater than $50 \%$, and an Observed/Expected $(\mathrm{O} / \mathrm{E})$ ratio of greater than 0.6 [3]. After Takai and Jones re-evaluated the three parameters in the GGF definition

\footnotetext{
* Correspondence: chyang@cc.kuas.edu.tw

${ }^{3}$ Department of Electronic Engineering, National Kaohsiung University of

Applied Sciences, Kaohsiung, 80778, Taiwan

Full list of author information is available at the end of the article
}

of $\mathrm{CpG}$ islands, they proposed a new set of criteria (GC

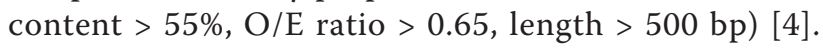
This algorithm can effectively exclude false positives from short repetitive sequences (e.g., Alu) [5].

Recently, various tools and methods have been proposed to predict CpG islands, e.g., CpGPlot [6], CpGProD [7], CpGIS [8] and CpGcluster [9], and the epigenome prediction method [10]. Rice et al. proposed the CpGPlot program, which plots CpG-rich areas and reports all CpG-rich regions [6]; CpGPlot yields the lowest sensitivity values. This could indicate that CpGPlot does not predict any islands in the target sequence and does not achieve a good performance. Ponger et al. proposed CpGProD, an application for identifying mammalian promoter regions associated with $\mathrm{CpG}$ islands in large genomic sequences. CpGProD shows the predicted probability over a transcription start site (TSS) chart, and the mammalian promoter regions associated with $\mathrm{CpG}$ island information [7]. CpGProD uses a sliding window technique, where the size of the window greatly influences the results, thus making its prediction unreliable [11]. Takai and Jones proposed the CpGIS algorithms, which merges two CpG islands when they were less than $100 \mathrm{bp}$ apart and the merged CpG island still mets the GGF criteria [8]. CpGIS provides a graphical map of the CpG dinucleotide distribution. Hackenberg et al. proposed CpGcluster, which uses only integers for 
calculations. It is thus a fast and efficient method for predicting significant clusters of $\mathrm{CpG}$ dinucleotides [9]. Bock et al. proposed an epigenome prediction method, which derives quantitative scores of "CpG island strength" that incorporate epigenetic and functional aspects to help resolve the problem that some current $\mathrm{CpG}$ island criteria incur significant disadvantages [10].

In this study, we present a web-based application called CpGPAP (CpG island predictor analysis platform) which uses the complementary particle swarm optimization (CPSO) [12] and a complementary genetic algorithm (CGA) [13] in combination with some methods from the literature. CPGPAP produces graphic visualizations of the $\mathrm{GC} \%$, the $\mathrm{O} / \mathrm{E}$ ratio, the distribution of $\mathrm{CpG}$ and the probability of a CpG island overlapping with a transcription start site. Furthermore, the proposed method allows users to freely select parameter settings and easily view $\mathrm{CpG}$ island information. This predictor platform can be of assistance to biologists involved in the study of $\mathrm{CpG}$ islands.

\section{Implementation}

The proposed CpGPAP interface was developed in JSP with Java script (jdk1.6.0_071.4.0). It is freely available at http://140.127.113.93/CpGPAP/.

\section{Results}

\section{Input module}

The input module of the CpGPAP platform contains three main functions. First, the prediction algorithm, i.e., CPSO, CGA, CpGPlot, CpGProD or CpGIS is selected (Figure 1A). Then, the optimization algorithm's parameters are set, which include algorithm-related and CpG island-related parameters ( $\mathrm{CpG}$ island length, GC content and $\mathrm{O} / \mathrm{E}$ ratio). FASTA sequences with the four nucleotides adenine $(A)$, thymine $(T)$, cytosine $(C)$ and guanine $(G)$ are accepted as input sequences (Figure 1B).

\section{Analysis module}

Figure 2 shows the optimization algorithm parameter settings. Subsequently, the predicted CpG island results, including the number of $\mathrm{CpG}$ islands, the $\mathrm{CpG}$ island start and end positions, the length of the $\mathrm{CpG}$ island, the $\mathrm{GC}$ content and the $\mathrm{O} / \mathrm{E}$ ratio, are shown. The "show chart" function can be used to further explore the results. The function visualizes the $\mathrm{CpG}$ island mapping, and depicts the CpG dinucleotides distribution, the GC content, the $\mathrm{O} / \mathrm{E}$ ratio distribution, and the $\mathrm{CpG}$ island sequence distribution.

\section{Output module}

To clearly determine the distribution of CpG islands, the CpGPAP platform generates a graphic visualization once the $\mathrm{CpG}$ islands prediction results are complete.
The design of the chart is mainly based on the GGF criteria; we thus focused on $\mathrm{GC}$ content (GC\%), O/E ratio and $\mathrm{CpG}$ island length design. The prediction results can be divided into four main types of CpG islandrelated information. (1) GC\% charts are calculated from the input sequence with a calculation processed every $50 \mathrm{bp}$ on average; (2) $\mathrm{O} / \mathrm{E}$ ratio charts are calculated through the same process as GC\%; (3) the predicted probability of being over the transcription start site chart is obtained by providing the CpGProD (http://pbil. univ-lyon1.fr/software/cpgprod.html); and (4) the distribution of $\mathrm{CpG}$ charts shows the predicted $\mathrm{CpG}$ islands resulting in the predicted genome sequence position, including the $\mathrm{CpG}$ island overlap input sequence position, the number of $\mathrm{CpG}$ islands and all connections to the $\mathrm{CpG}$ nucleic position. All of the above results are shown in Figure 3. Theoretically, in the large-scale computational analysis of CpG islands, the CpGPAP platform can accept any sequence input and dataset size. However, to avoid data transfer errors, we limited the "show charts" function to display graphic sequence information of $50 \mathrm{~kb}$ or less. The graphic visualization allows researchers to set related parameters accurately and obtain better prediction results. A stand-alone version is also available for download with no input sequence size limitation.

\section{Related tools and dataset download}

The CpGPAP platform also includes CpGPlot, CpGProD, CpGIS, and CpGcluster all of which are related $\mathrm{CpG}$ island prediction tools. We furthermore included dataset links for related downloads, including the National Center for Biotechnology Information (NCBI), the University of California Santa Cruz (UCSU), RegulonDB, the Database of Transcriptional Start Sites (DBTSS), and the European Bioinformatics Institute (EMBL-EBI) for the users convenience.

\section{Discussion}

DNA methylation is a major epigenetic modification of the genome that affects basic biological functions [14] such as the gene expression [15] and human diseases, especially cancer $[16,17]$. DNA methylation occurs most commonly in a $\mathrm{CpG}$ sequence called $\mathrm{CpG}$ islands. Hence, we developed a visualization tool that depicts CpG island distribution charts to facilitate methylation related studies.

Several methods are already available to determine a CpG island region, e.g., CpGPlot, CpGProD, CpGIS, and CpGcluster. However, these methods, with the exception of CpGIS, do not simultaneously provide the GC content, the $\mathrm{O} / \mathrm{E}$ ratio distribution and the $\mathrm{CpG}$ islands sequence distribution. A comparison of currently used platforms is shown in Table 1. The CpGPlot, CpGProD, CpGIS, and 


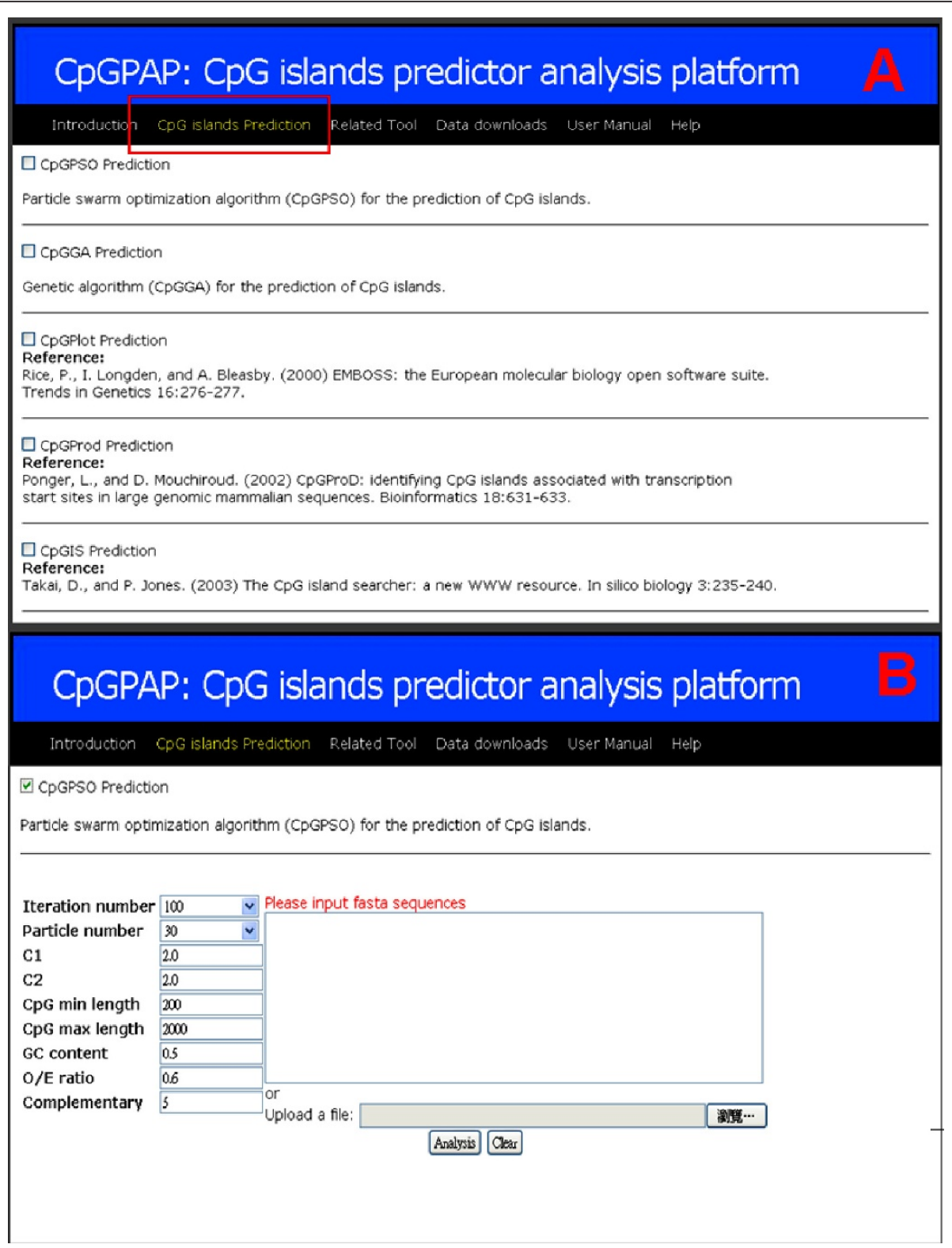

Figure 1 CpGPAP platform flowchart. A: Selection of the optimization algorithm for predicting CpG islands. B: Parameter settings for the optimization algorithm, $\mathrm{CpG}$ island related parameters and input sequence

CpGcluster methods used to predict CpG islands are discussed in the methods section. These tools, with the exception of CpGcluster, use methods that are based on three parameters, namely the GC content, the $\mathrm{O} / \mathrm{E}$ ratio and the $\mathrm{CpG}$ island length, to predict $\mathrm{CpG}$ islands. Most use the sliding window technique with the GC content, the $\mathrm{O} / \mathrm{E}$ ratio and length thresholds as the main parameters, while CpGcluster uses the distance between CpG dinculeotides. The sliding window technique is similar to brute force searches and thus yields high SP values. Han et al. $[18,19]$ and Hackenberg et al. $[9,20]$ identified several disadvantages of methods based on the sliding window technique: (1) CpG islands identified by these methods generally do not start and end with a CpG dinucleotide [21]. (2) The number and length of the CpG islands is obtained based on the window size and the step size. If the window is large, several short and loosely distributed CpG islands may be merged into a larger one. (3) The run time for these methods is relatively long. The CpGcluster method predicts CpG islands based on the physical distance between CpG dinucleotides [20]. Although CpGcluster can identify some short CpG islands, a high number of false positives $(F P)$ is obtained over the entire genome [18]. In addition, other problems 


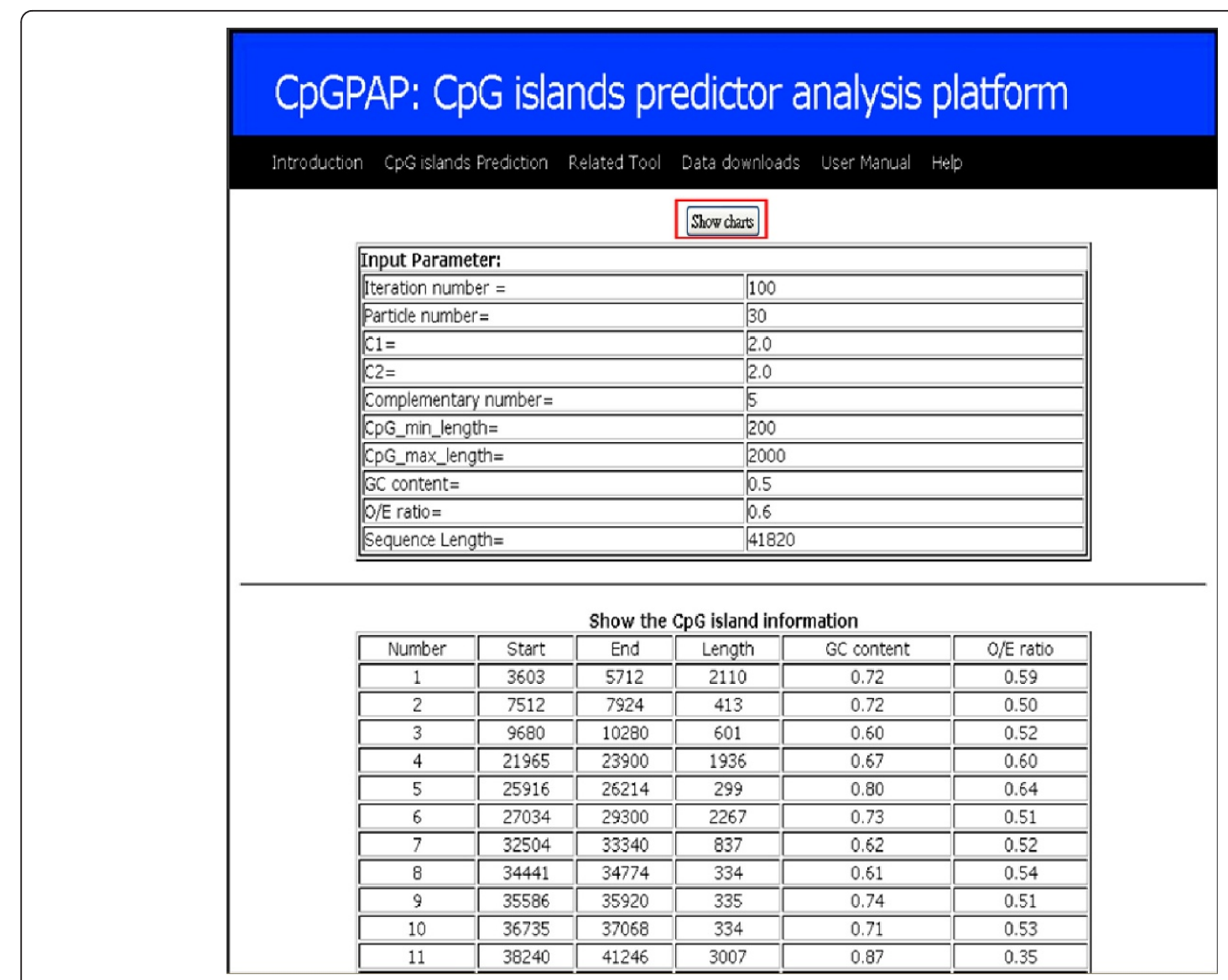

Figure 2 Prediction results showing CpG island-related information. such as number of CpG islands, CpG island length, start and end position, input parameters, O/E ratio, and GC content

exist for CpGCluster: (1) Search results are dependent on the composition of the sequence scanned, i.e., a CpG island identified in one sequence may be discarded when planted into another sequence with a different composition. (2) It also has a low prediction sensitivity [9]. This shows that there is still room for improvement.

In order to validate the performance of the complementary PSO (CPSO) and complementary GA (CGA) (see Additional file 1: Algorithm CGA), i.e., the two algorithms supported by our platform; we compared them to other methods on the human chromosomes 21 and 22 . Chromosomes 21 and 22 are widely used in the literature, so we used the available data on the chromosomes for the validation process. Calculations for all the other human chromosomes can be found in [22]. Table 2 shows a comparison to the other methods. Information includes the chromosome length, the total length of the $\mathrm{CpG}$ islands, the number of islands predicted, the coverage (\%), the island length (average, minimum, and maximum), the GC content, and $\mathrm{CpG}$ island $\mathrm{O} / \mathrm{E}$ ratio values. Table 2 indicates that the number of $\mathrm{CpG}$ islands predicted by CpGIS is the highest for chromosomes 21
$(3,704)$ and $22(6,875)$. However, the total number of CpG islands does not represent a better prediction ability of this method since the average length of $\mathrm{CpG}$ islands predicted by CpGIS (346 bp and $413 \mathrm{bp}$ for chromosome 21 and 22, respectively) is shorter than in our supported algorithms CPSO obtained a result of 571 bp and 596 bp for chromosomes 21 and 22, respectively. CGA obtained $482 \mathrm{bp}$ and $619 \mathrm{bp}$ for chromosomes 21 and 22, respectively. This indicates that the total length of the CpG islands predicted in chromosomes 21 and 22 by CpGIS is shorter than the length predicted by CPSO and CGA. In addition, when compared to the methods from the literature, the islands predicted by CPSO covered a larger region (3.4\% and 5.85\%) in chromosomes 21 and 22, respectively. The percentages of the region covered by CGA are $3.39 \%$ and $6.20 \%$ in chromosomes 21 and 22, respectively. The supported algorithms' average values for the GC content, the $\mathrm{O} / \mathrm{E}$ ratio and the length of the predicted $\mathrm{CpG}$ islands all conform to the GGF criteria.

In general, around $80 \%$ of all CpG dinucleotides are methylated in mammalian genomes [23]. The lack of 


\section{CpGPAP: CpG islands predictor analysis platform}

Introduction CPGislands Prediction Related Tool Data downloads User Manual Help

A

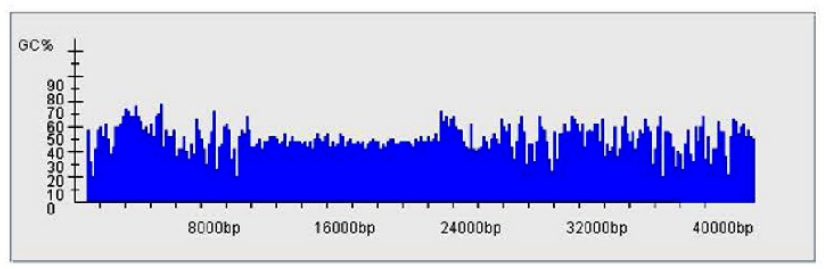

B

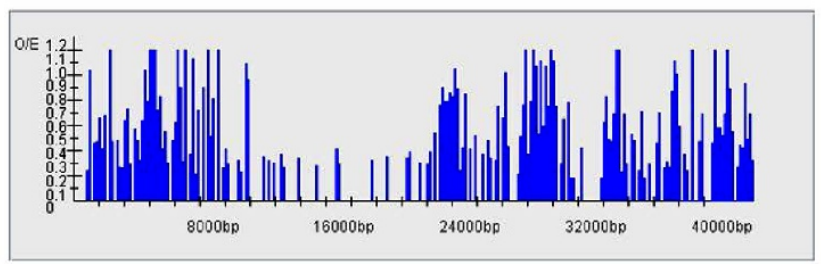

C

Predicted probability to be located over the transcription start site
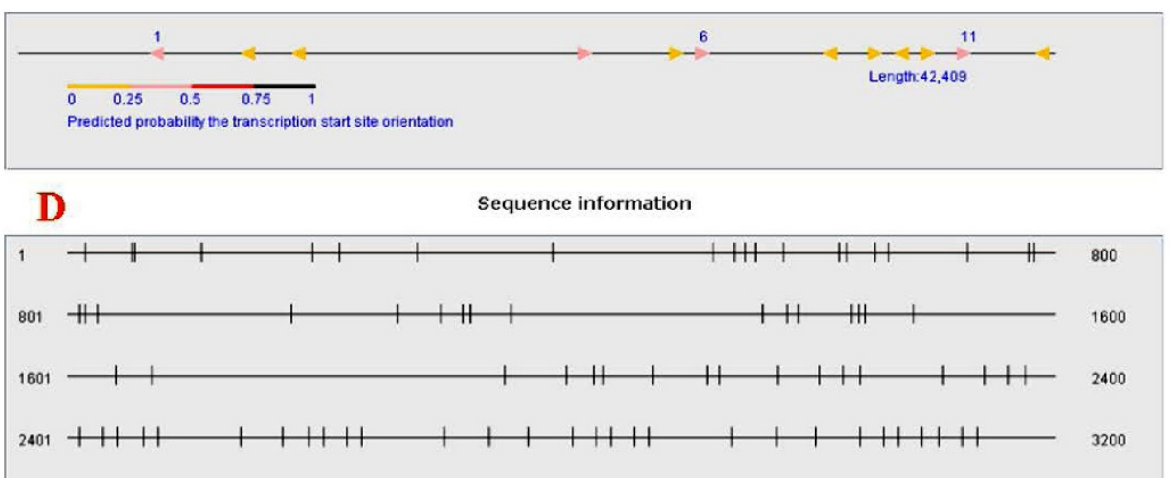

Figure 3 Visualization of the CpG island prediction results. A: GC\% chart shows the GC content distribution in the input sequence. B: O/E ratio chart shows the O/E ratio distribution in the input sequence. C: TSS chart shows the probability of the predicted CpG island overlapping with a transcription start site. D: $\mathrm{CpG}$ chart shows the $\mathrm{CpG}$ nucleic and $\mathrm{CpG}$ island distribution

methylation is thus a very good indicator of the function of a CpG island [19,22]. The analysis platform was used to investigate the CpG island distribution in a DNA genome and obtained better prediction results for CpG islands than the other methods it was compared to. The accuracies $(A C C)$ of the supported algorithms were also higher [24].

\section{Advantages of CpGPAP}

CPGPAP has several advantages over other analysis platforms. Firstly, the platform-supported algorithms reach a high correlation coefficient $(C C)$ (See Additional file 1: Table S1, Table S2 and Table S3). Secondly, CpGPAP provides an easily accessible interface for convenient visualization and analysis of genomic CpG islands. Furthermore, CpGPAP integrates currently existing tools and provides datasets for users to download. The supported algorithms (CPSO and CGA) predict CpG islands faster than other methods. The platform also provides a graphical overview of the putative islands. A stand-alone version with no limitations on the input sequence length is also available. This stand-alone version includes a visual display function. CpGPAP is thus a very useful tool for the detection and analysis of genomic CpG islands.

\section{Conclusions}

We propose a novel CpG island prediction platform, CpGPAP, in which the platform's supported algorithms (CPSO and CGA) provide a higher sensitivity $(S N)$ and a correlation coefficient $(C C)$ as compared to the CpGPlot, CpGProD, CpGIS and CpGcluster platforms over an entire chromosome. CPGPAP integrates relevant 
Table 1 Comparison of different software functions for CpG island

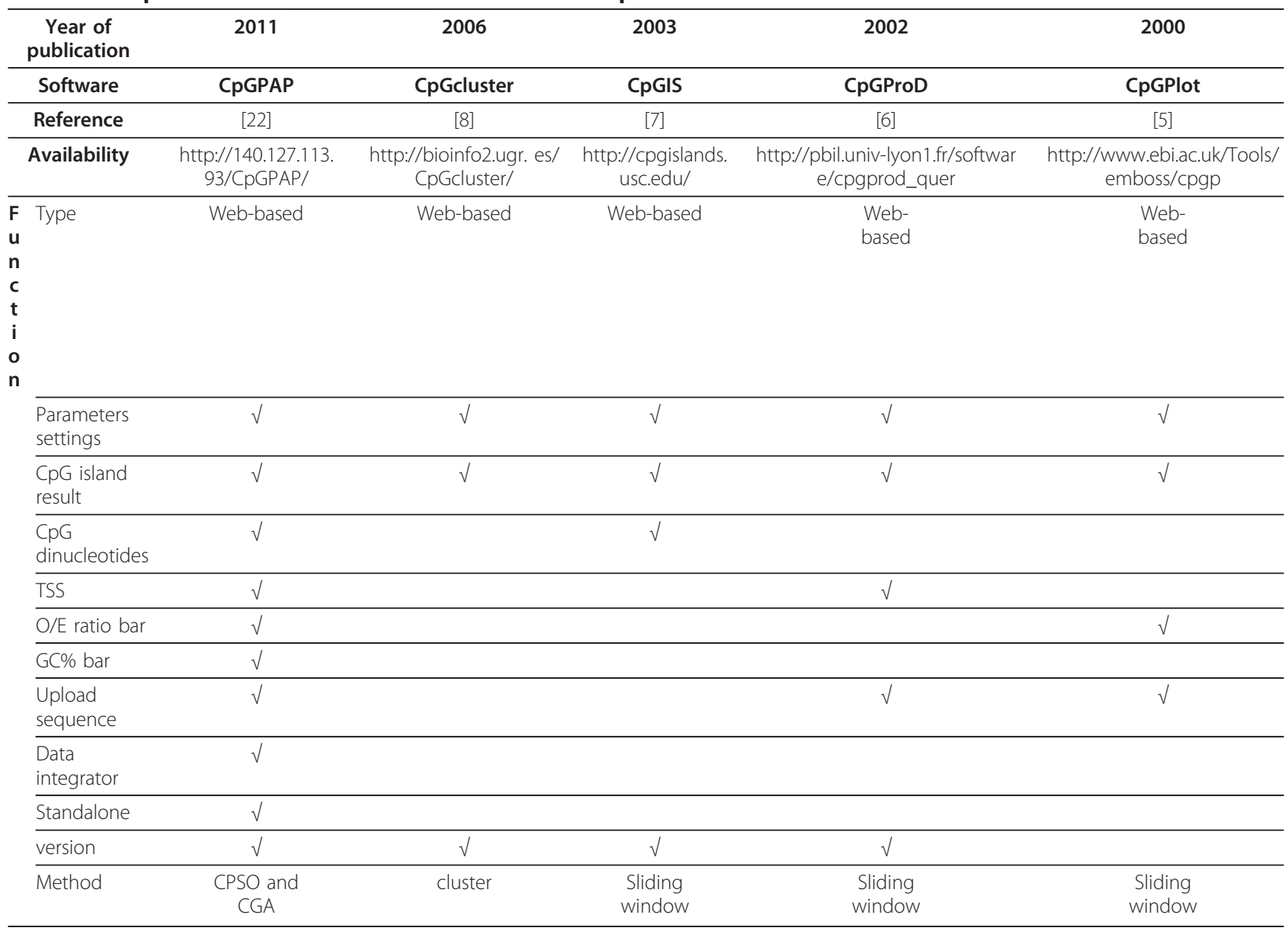

TSS: transcription start site. CPSO: complementary particle swarm optimization. CGA: complementary genetic algorithm

approaches from the literature to provide the user with more options. The incorporated algorithms (CPSO and CGA) have a lower computational complexity than the other platforms in the literature. We integrated the three related prediction tools CpGPlot, CpGProD and CpGIS and provided dataset links for easy access. The $\mathrm{CpG}$ island prediction parameters can be selected freely. We believe that the proposed predictor platform can be of assistance to biologists involved in the study of $\mathrm{CpG}$ islands.

\section{Methods}

\section{Supported algorithms}

To predict $\mathrm{CpG}$ islands, the CpGPAP analysis platform includes two separate algorithms, CPSO and CGA. It also incorporates internet links to other prediction methods, namely CpGIS, CpGPlot and CpGProD. The development of the analysis platform is a continuation of our previous CpG island study [24].

PSO is a population-based stochastic optimization algorithm, which was developed by simulating the social behavior of organisms [12]. In PSO, each particle in the search space can be considered to be an individual bird in a flock, which changes its position based on its memory and its knowledge of its neighbors. Each particle from a swarm represents a candidate solution. The individual best value $\left(\right.$ pbest $\left._{i}\right)$ is the position of the $i$-th particle with the highest fitness at a given iteration; the best position of all pbest is called gbest. Particles use their individual memory (pbest) and the swarm's knowledge (gbest) as a whole to move around a multidimensional search space until the termination condition is reached. PSO has been successfully applied in many fields, including operon [25] and CpG island prediction [24], amongst others. The pseudo-code of PSO for the prediction CpG island is shown in Additional file 1: Figure S1.

GA is a stochastic search algorithm modeled after the process of natural selection that underlies biological evolution [13]. The standard GA procedure applies the following genetic operators: chromosome encoding and initialization, selection, crossover and mutation, which is the process by which a whole generation of new offsprings is computed. By applying genetic operators on strings in the mating pool, a new population of strings is formed in the next generation. The implementation of 
Table 2 Comparison of the number of CpG islands identified in the human genome with different methods (NCBI.36)

\begin{tabular}{|c|c|c|c|c|c|c|c|c|}
\hline & \multicolumn{7}{|c|}{ Chromosome 21} & \multirow[b]{2}{*}{$C G A$} \\
\hline & CpGPlot & CpGcluster & CpGProD & CpGIS & PSO & CPSO & GA & \\
\hline Chromosome Length (bp) & & & & $46,944,329$ & & & & \\
\hline Total length of $\mathrm{CpG}$ islands & 347,334 & 639,161 & $1,072,192$ & $1,280,505$ & $1,564,596$ & $1,607,472$ & $1,262,449$ & $1,589,629$ \\
\hline Number of islands predicted & 973 & 2,703 & 1,091 & 3,704 & 2,648 & 2,813 & 2,513 & 3,304 \\
\hline Island coverage $(\%)$ & 0.73 & 1.36 & 2.28 & 2.73 & 3.3 & 3.4 & 2.68 & 3.39 \\
\hline \multicolumn{9}{|l|}{ Island length (bp) } \\
\hline Average & 357 & 237 & 983 & 346 & 591 & 571 & 502 & 482 \\
\hline Minimum & 101 & 8 & 500 & 200 & 202 & 202 & 201 & 201 \\
\hline Maximum & 3,047 & 3,028 & 6,732 & 1,948 & 4,020 & 4,035 & 6,126 & 10,687 \\
\hline GC-content \pm SD (\%) & $62.17 \pm 0.07$ & $65.49 \pm 0.07$ & $54.49 \pm 0.06$ & $57.98 \pm 0.04$ & $53.73 \pm 0.05$ & $53.72 \pm 0.05$ & $54.24 \pm 0.05$ & $55.07 \pm 0.05$ \\
\hline \multirow[t]{3}{*}{$\mathrm{CpG}$ island $\mathrm{O} / \mathrm{E}$ ratio $\pm \mathrm{SD}$} & $0.84 \pm 0.1$ & $0.87 \pm 0.3$ & $0.63 \pm 0.1$ & $0.68 \pm 0.1$ & $0.64 \pm 0.08$ & $0.65 \pm 0.08$ & $0.68 \pm 0.1$ & $0.71 \pm 0.1$ \\
\hline & \multicolumn{8}{|c|}{ Chromosome 22} \\
\hline & CpGPlot & CpGcluster & CpGProD & CpGIS & PSO & CPSO & $G A$ & $C G A$ \\
\hline Chromosome Length (bp) & \multicolumn{8}{|c|}{$49,691,432$} \\
\hline Total length of CpG islands & 679,803 & 522,748 & $2,067,653$ & $2,842,255$ & $2,802,675$ & $2,907,983$ & $2,251,454$ & $3,085,715$ \\
\hline Number of islands predicted & 1,642 & 2,186 & 1,903 & 6,875 & 4,571 & 4,882 & 3,902 & 4,985 \\
\hline Island coverage (\%) & 1.36 & 1.05 & 4.16 & 5.71 & 5.64 & 5.85 & 4.53 & 6.20 \\
\hline \multicolumn{9}{|l|}{ Island length (bp) } \\
\hline Average & 414 & 239 & 1,087 & 413 & 613 & 596 & 577 & 619 \\
\hline Minimum & 200 & 8 & 500 & 200 & 198 & 202 & 201 & 201 \\
\hline Maximum & 7,902 & 7,774 & 8,363 & 3,339 & 4,076 & 4,076 & 5,340 & 5,905 \\
\hline GC-content \pm SD (\%) & $63.70 \pm 0.08$ & $70.23 \pm 0.08$ & $55.84 \pm 0.07$ & $55.12 \pm 0.06$ & $54.50 \pm 0.07$ & $54.46 \pm 0.07$ & $55.21 \pm 0.05$ & $56.15 \pm 0.06$ \\
\hline $\mathrm{CpG}$ island $\mathrm{O} / \mathrm{E}$ ratio $\pm \mathrm{SD}$ & $0.84 \pm 0.1$ & $0.95 \pm 0.3$ & $0.62 \pm 0.1$ & $0.68 \pm 0.1$ & $0.63 \pm 0.05$ & $0.63 \pm 0.05$ & $0.64 \pm 0.1$ & $0.68 \pm 0.1$ \\
\hline
\end{tabular}

SD: Standard Deviation. Proportion (\%) of the chromosome sequence covered by methods

the genetic operators is repeated in each subsequent generation until a termination condition is reached. GAs have been successfully applied in many fields, e.g. microarray data exploration [26] and SNP interaction studies [27]. The pseudo-code of the GA for CpG island prediction is shown in Additional file 1: Figure S2.

If the PSO and GA search processes fall into a local optimum for five consecutive generations, the complementary concept is used to leave this local region and re-enter the global search. Additional file 1: Figure S3 shows the flowchart of the complementary GA (CGA), and Additional file 1: Figure S4 shows the flowchart of the complementary PSO (CPSO) as well as an illustrative example of how the CGA algorithm works. In addition, we integrated three sliding windows methods (CpGIS, CpGPlot and CpGProD) in the CpGPAP platform to predict $\mathrm{CpG}$ islands. These methods use the GC content, the $\mathrm{O} / \mathrm{E}$ ratio and $\mathrm{CpG}$ island length as the main parameters to predict $\mathrm{CpG}$ islands.

\section{System overview}

Using the CpGPAP platform involves three steps. First, users select the optimization algorithms used to predict the CpG islands (Figure 1A). Then, the optimization algorithm's parameters and CpG island related parameters are set and the input sequence is uploaded (Figure 1B). In a final step, CpG island-related information predicted by the algorithm, such as length, start and end position, input parameters, O/E ratio, GC content, etc., is display (Figure 2). Users can choose whether to display a visualization of the prediction results (Figure 3), and CpGPAP parameters can be freely modified. Figure 4 shows the structure and flowchart of the CpGPAP system. In addition, while the other algorithms were initially designed based on the GGF criteria (i.e., GC content $\geq 50 \%, \mathrm{O} / \mathrm{E}$ ratio $\geq 0.6$, and $\mathrm{CpG}$ island length $>200 \mathrm{bp}$ ), the parameters related to CpG islands can be freely modified in CPGPAP.

\section{Availability and requirements}

Project home page: http://140.127.113.93/CpGPAP/

Operating system(s): Operating systems with web browser.

Programming language: Java, javascript, CSS and HTML.

Other requirements: Java 1.5.0 (or later).

License: none for academic users. For any restrictions regarding the use by non-academics please contact the corresponding author. 


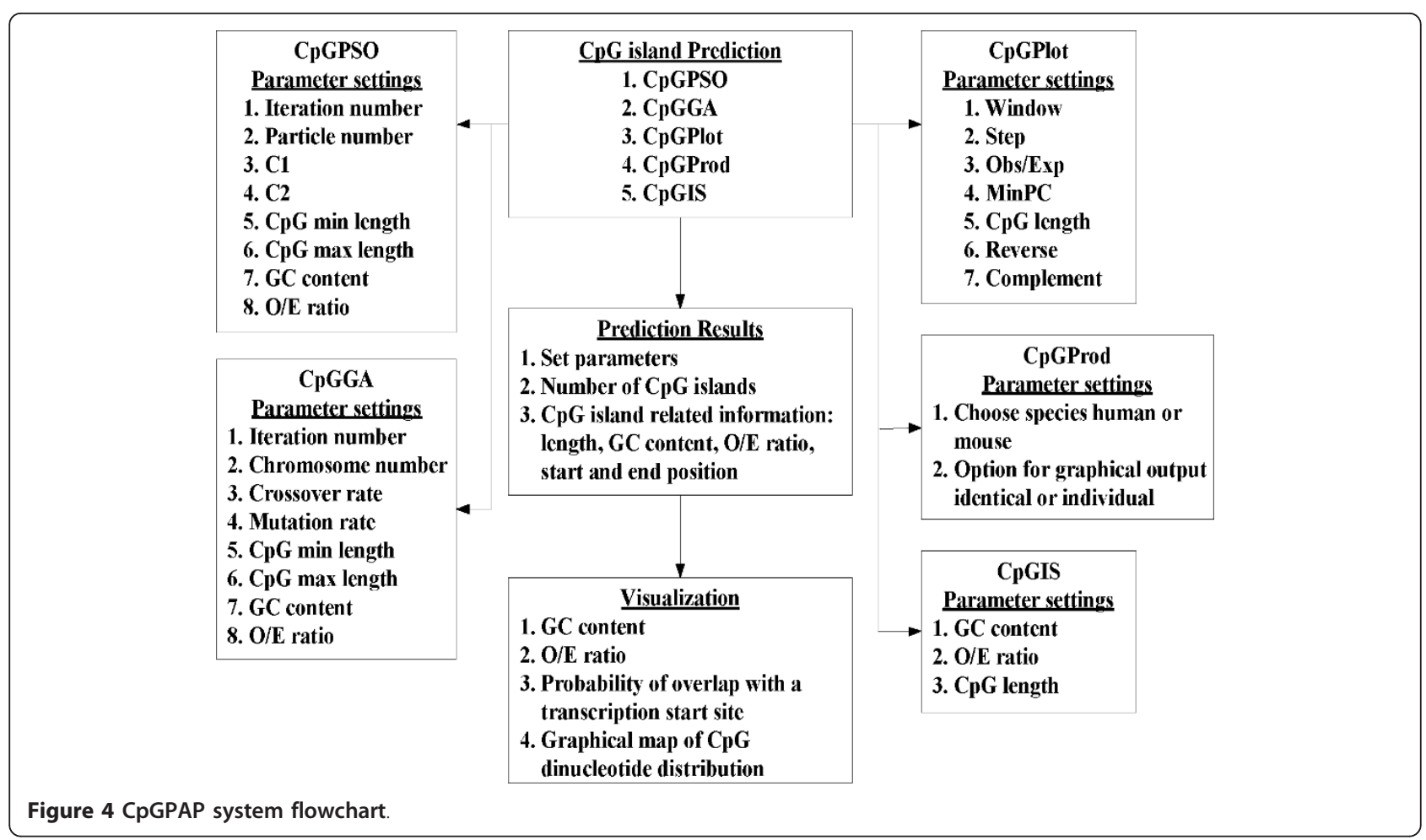

\section{Additional material}

Additional file 1: Table S1. Comparison of different methods for CpG island prediction (CPSO). Table S2. Comparison of different methods for CpG island prediction (CpGGA). Table S3. Comparison of different methods on the number of $\mathrm{CpG}$ islands identified in the entire human genomes. Supplementary Methods. Algorithm CGA. Figure S1. The pseudo-code for the PSO. Figure S2. The pseudo-code for the GA. Figure S3. Flowchart for the complementary GA. Figure S4. Flowchart for the complementary PSO $[3,13,28,29]$.

\section{Acknowledgements}

This work is partly supported by Taiwan's National Science Council under grants NSC96-2221-E-214-050-MY3, NSC98-2221-E-151-040-, NSC 98-2622-E151-001-CC2, 98-2622-E-151-024-CC3, NSC 99-2622-E-151-019-CC3 and NSC 99-2221-E-151-056-.

\section{Author details}

${ }^{1}$ Department of Chemical Engineering \& Institute of Biotechnology and Chemical Engineering, I-Shou University, Kaohsiung, 8004, Taiwan. ${ }^{2}$ Department of Electronic Communication Engineering, National Kaohsiung Marine University, Kaohsiung, 81157, Taiwan. ${ }^{3}$ Department of Electronic Engineering, National Kaohsiung University of Applied Sciences, Kaohsiung,

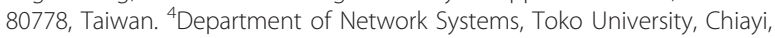
61363, Taiwan.

\section{Authors' contributions}

LYC conceived of the study and drafted the manuscript. CHY participated in the design and helped to draft the manuscript. MCL participated in the design of the study and performed the analysis. CHY participated in its design and coordination and helped to draft the manuscript. All authors read and approved the final manuscript.

Received: 20 August 2011 Accepted: 2 March 2012 Published: 2 March 2012
References

1. Feil $R$, Berger F: Convergent evolution of genomic imprinting in plants and mammals. Trend Genet 2007, 23(4):192-199.

2. Tykocinski M, Max E: CG dinucleotide clusters in MHC genes and in 5'demethylated genes. Nucleic Acids Res 1984, 12(10):4385.

3. Gardiner-Garden M, Frommer M: CpG Islands in vertebrate genomes* 1. J Mol Biol 1987, 196(2):261-282.

4. Takai D, Jones PA: Comprehensive analysis of CpG islands in human chromosomes 21 and 22. Proc Natl Acad Sci USA 2002, 99(6):3740-3745.

5. Schmid CW: Does SINE evolution preclude Alu function? Nucleic Acids Res 1998, 26(20):4541-4550.

6. Rice P, Longden I, Bleasby A: EMBOSS: the European molecular biology open software suite. Trends Genet 2000, 16(6):276-277.

7. Ponger L, Mouchiroud D: CpGProD: identifying CpG islands associated with transcription start sites in large genomic mammalian sequences. Bioinformatics 2002, 18(4):631

8. Takai D, Jones P: The CpG island searcher: a new WWW resource. In Silico Biol 2003, 3(3):235-240.

9. Hackenberg M, Previti C, Luque-Escamilla P, Carpena P, Martinez-Aroza J, Oliver J: CpGcluster: a distance-based algorithm for CpG-island detection. BMC Bioinforma 2006, 7(1):446.

10. Bock C, Walter J, Paulsen M, Lengauer T: CpG island mapping by epigenome prediction. PloS Comput Biol 2007, 3(6):1055-1070.

11. Luque-Escamilla P, Martinez-Aroza J, Oliver J, Gomez-Lopera J, RomanRoldan R: Compositional searching of CpG islands in the human genome. Phys Rev E 2005, 71(6):61925.

12. Kennedy J, Eberhart R: Particle swarm optimization. IEEE International Conference on Neural Networks: 1995 1995, 1942-1948.

13. John H: Adaptation in Nature and Artificial Systems University of Michigan Press; 1975, 120-142.

14. Kienesberger $P C$, Oberer M, Lass A, Zechner R: Mammalian patatin domain containing proteins: a family with diverse lipolytic activities involved in multiple biological functions. J Lipid Res 2009, 50:S63-S68.

15. Hyde SC, Pringle IA, Abdullah S, Lawton AE, Davies LA, Varathalingam A, Nunez-Alonso G, Green AM, Bazzani RP, Sumner-Jones SG: CpG-free plasmids confer reduced inflammation and sustained pulmonary gene expression. Nat Biotechnol 2008, 26(5):549-551. 
16. Christensen BC, Houseman EA, Marsit CJ, Zheng S, Wrensch MR, Wiemels JL, Nelson HH, Karagas MR, Padbury JF, Bueno R: Aging and environmental exposures alter tissue-specific DNA methylation dependent upon CpG island context. PLoS Genet 2009, 5(8):e1000602.

17. Harder J, Engelstaedter V, Usadel H, Lassmann S, Werner M, Baier P, Otto F, Varbanova M, Schaeffner E, Olschewski M: CpG-island methylation of the ER promoter in colorectal cancer: analysis of micrometastases in lymph nodes from UICC stage I and II patients. Br J Cancer 2009, 100(2):360-365.

18. Han $\mathrm{L}$, Zhao Z: CpG islands or CpG clusters: how to identify functional GC-rich regions in a genome? BMC Bioinforma 2009, 10(1):65.

19. Zhao Z, Han L: CpG islands: algorithms and applications in methylation studies. Biochem Biophys Res Commun 2009, 382(4):643-645.

20. Hackenberg M, Barturen G, Carpena P, Luque-Escamilla P, Previti C, Oliver J: Prediction of $\mathrm{CpG}$-island function: $\mathrm{CpG}$ clustering vs. sliding-window methods. BMC Genomics 2010, 11(1):327.

21. Lai H, Chiang Y, Hsu C, Wu F: A recognition machine for CpG-islands based on Boltzmann model. J Med Biol Eng 2008, 28(1):23-30.

22. Illingworth R, Bird A: CpG islands-A' rough guide'. FEBS Lett 2009, 583(11):1713-1720.

23. Yegnasubramanian S, Haffner MC, Zhang Y, Gurel B, Cornish TC, Wu Z, Irizarry RA, Morgan J, Hicks J, DeWeese TL, et al: DNA hypomethylation arises later in prostate cancer progression than $\mathrm{CpG}$ island hypermethylation and contributes to metastatic tumor heterogeneity. Cancer Res 2008, 68(21):8954-8967.

24. Chuang L-Y, Huang H-C, Lin M-C, Yang C-H: Particle Swarm Optimization with Reinforcement Learning for the Prediction of $\mathrm{CpG}$ Islands in the Human Genome. PLoS One 2011, 6(6):e21036.

25. Chuang $L Y$, Tsai $J H$, Yang CH: Binary particle swarm optimization for operon prediction. Nucleic Acids Res 2010, 38(12):e128

26. Peng $S, X u Q$, Ling XB, Peng X, Du W, Chen L: Molecular classification of cancer types from microarray data using the combination of genetic algorithms and support vector machines. FEBS Lett 2003, 555(2):358-362

27. Yang $C H$, Chuang LY, Chen YJ, Tseng HF, Chang HW: Computational analysis of simulated snp interactions between 26 growth factor-related genes in a breast cancer association study. OMICS 2011, 15(6):399-407.

28. Fang F, Fan S, Zhang $X$, Zhang MQ: Predicting methylation status of $\mathrm{CpG}$ islands in the human brain. Bioinformatics 2006, 22:2204-2209.

29. Whitehead S, Sutton R, Ballard D: Advances in reinforcement learning and their implications for intelligent control. Proceedings of the 5th IEEE Int Symposium on Intelligent Control 1990, 289-1297.

doi:10.1186/1471-2156-13-13

Cite this article as: Chuang et al: CpGPAP: CpG island predictor analysis platform. BMC Genetics 2012 13:13.

\section{Submit your next manuscript to BioMed Central and take full advantage of:}

- Convenient online submission

- Thorough peer review

- No space constraints or color figure charges

- Immediate publication on acceptance

- Inclusion in PubMed, CAS, Scopus and Google Scholar

- Research which is freely available for redistribution

Submit your manuscript at www.biomedcentral.com/submit
Biomed Central 